\title{
The Dexterity Management for Medical Spare Parts Archives
}

\author{
Xin Mai', Zhifeng Ye1, Zhe Wei², Changhua Chen'1, Haili Ding1, Ran Wang1 \\ ${ }^{1}$ Library, Nanjing University of Aeronautics and Astronautics, Nanjing, China \\ ${ }^{2}$ Department of Information, No. 940 Hospital of PLA's Joint Service Support Force, Lanzhou, China \\ Email: jiangwei_xm@aliyun.com
}

How to cite this paper: Mai, X., Ye, Z.F., Wei, Z., Chen, C.H., Ding, H.L. and Wang, R. (2019) The Dexterity Management for Medical Spare Parts Archives. Intelligent Information Management, 11, 33-37. https://doi.org/10.4236/iim.2019.112003

Received: January 9, 2019

Accepted: March 4, 2019

Published: March 7, 2019

Copyright (c) 2019 by author(s) and Scientific Research Publishing Inc. This work is licensed under the Creative Commons Attribution International License (CC BY 4.0).

http://creativecommons.org/licenses/by/4.0/

\begin{abstract}
In the field of medical equipment and equipment storage work, when we access accessories and equipment, due to the actual conditions, it causes low efficiency. It is often difficult to quickly and efficiently search by using simple data management software, such as excel. In order to solve the problem, our team used Python development tool to build high-performance retrieval software, which enabled the equipment custodian to maintain or redesign the database according to actual needs. Through actual verification, this system not only can greatly improve the accuracy of device access, but also has good expansion portability, which could manage various multimedia data systems.
\end{abstract}

\section{Keywords}

Archives, Python, Dexterity, Management

\section{Introduction}

In the field of medical engineering technology, especially in the actual work of equipment support, the requirements for the storage of spare parts are very high. It is no exaggeration to say that in some special cases, the ability to quickly retrieve a special spare part through file screening can determine the progress of a project [1]. With the current comprehensive advancement of informatization reform in the field of medical engineering technology, the contradictions encountered are also constantly highlighted [2].

When accessing spare parts, technicians rely on manual retrieval of various types of paper materials or simple classified electronic materials, which are mostly stored by simple data management software such as excel [3]. These file data exists many problems: single type, low scalability and low human-computer interaction. In actual work, it is often impossible to encounter parts with the 
same parameters [4], which will have different models or appearances, and the trouble caused by the wrong using is not uncommon.

In the context of the current popularity of small smart terminals, a smart retrieval system with powerful multimedia file management capabilities has become the expectation of the majority of technicians [5]. In order to solve those problems, the researchers of our group use Python development tools to design a multimedia data file management system to meet the actual needs of technicians and solve those problems.

\section{Construction of this System}

Due to the opening source of Python development tools, it is highly portable and can be used across platforms. It can be used not only in WINDOWS type systems [6], but also in systems including Android, which is based on LINUX system. The selection of the system gets great convenience and the operating platform is no longer limited in larger devices such as desktop computers and notebook computers [7]. Now a small tablet, even a smart-phone with a certain amount of storage space can run this system, which greatly enhances its dexterity.

The entire system is designed according to the flow shown in Figure 1, to achieve the corresponding retrieval and selection functions.

\section{Experimental Argumentation}

The tablet computer with the WIN10 system is installed as a hardware platform, and the developed system is operated. The interface shown in Figure 2 (front panel) is simple and friendly and it is convenient and practical with the help of touch-operation.

To demonstrate the specific functions of the system, the following is an example of retrieving screw parts. First, click the search path button in Figure 2 (front panel) to add the search folder path to the path library in Figure 2 (rear panel), in which you can add multiple folders as needed. After this setting, the system traverses the information in each of the folders by the selected search path.

The system can select different parameters for searching according to requirements. Therefore, even if you can't remember the specific name, etc., as long as you know a small number of parameters according to actual needs, you can also find the equipment that meets the corresponding conditions.

For example, according to the tooth option, we retrieve the screw with a tooth of $13 \mathrm{~mm}$. The system gives two card frames (shown in Figure 3), which are M1 13 flat-headed screw (copper) and M1 13 flat-headed screw (stainless steel).

The usage rules, drawings and video examples are all linked in the buttons in the corresponding card. Click the raised button below, you'll see the text version of the link's usage rules, the corresponding equipment icon and video explanation.

Then click the "Image" (图片) button of these two parameter cards respectively, then the corresponding physical pattern appears, which are shown in 


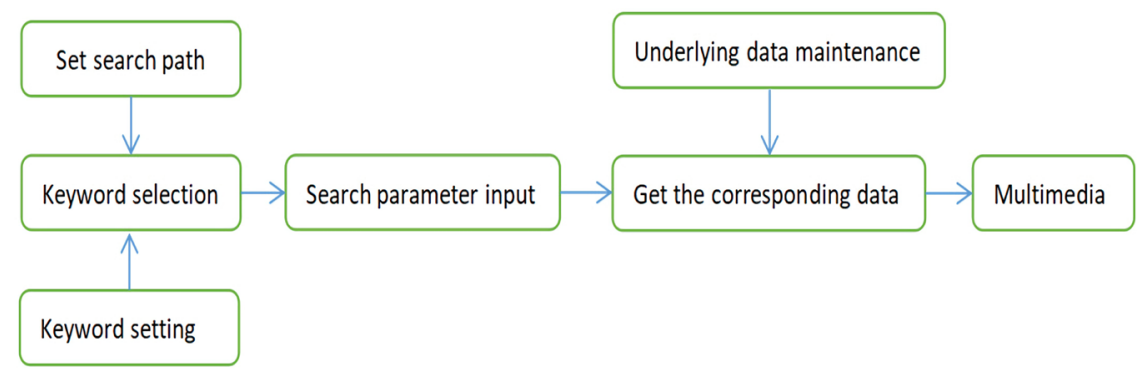

Figure 1. System operation process.

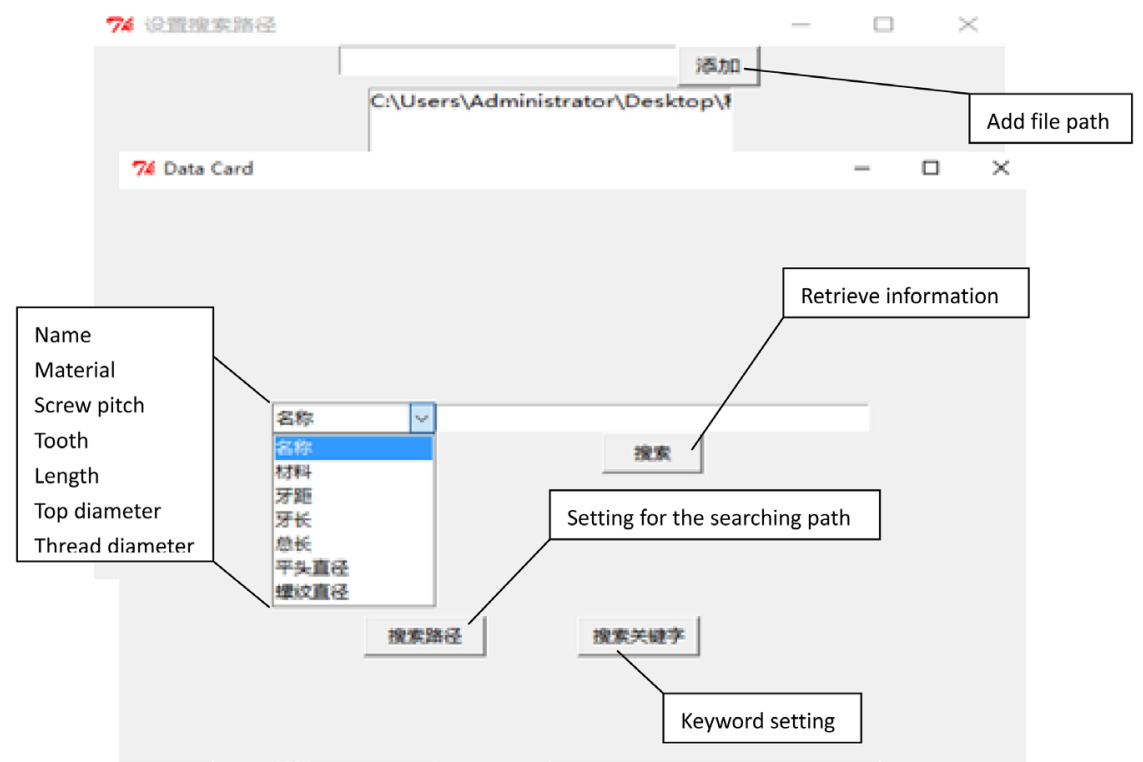

Figure 2. File retrieval path library (below) and search option diagram (above).

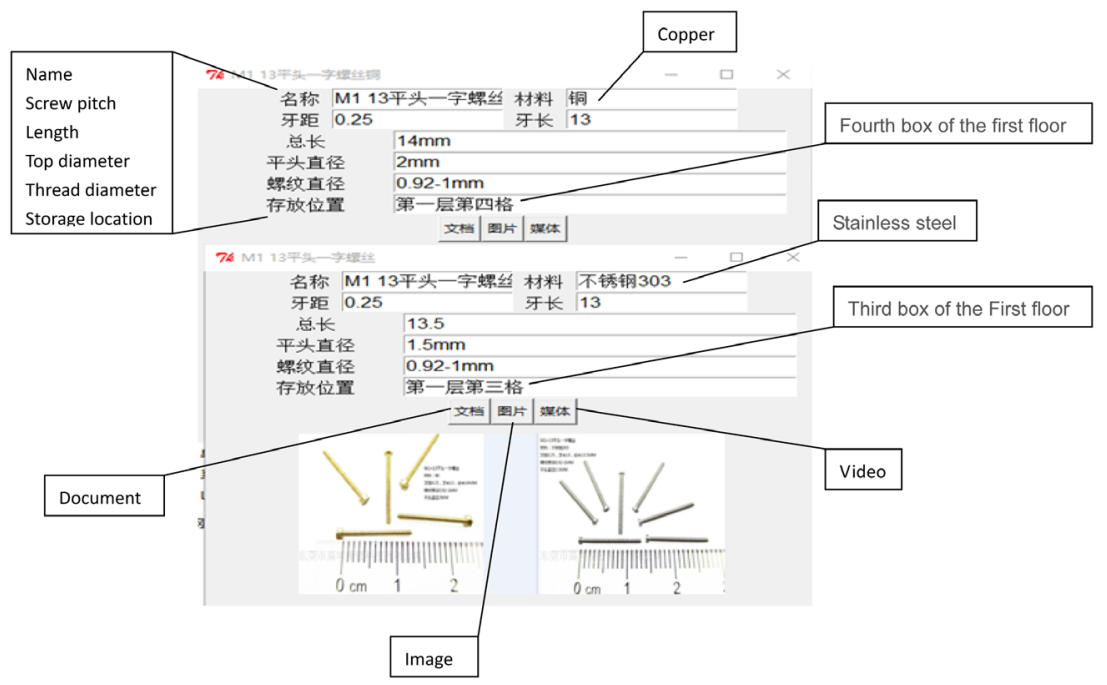

Figure 3. Searching results and buttons of links.

Figure 3. By looking at the picture, it is possible to accurately identify the required device and achieve precisely and efficiently accessing to the equipment. 


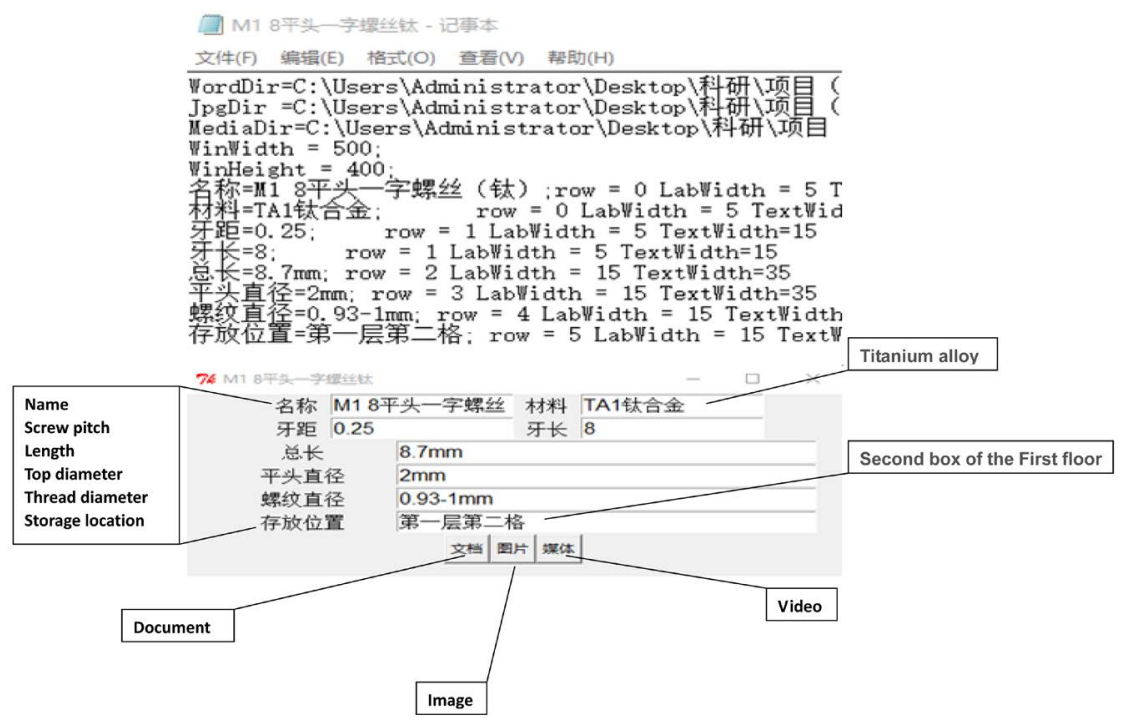

Figure 4. The underlying data to be retrieved (top) and the generated search card (bottom).

In order to realize the simplicity of the system, when setting the underlying data interface, the file of the Notepad-type is selected. When new equipment and retrieval parameters need to be added in the equipment library, just find the corresponding file in the system file package, open the "ini type" file, and then edit it in the form of a notepad file, as shown in Figure 4.

In Figure 4, the three lines of "WordDir", "JpgDir", and "MediaDir" in the Notepad file correspond to the file paths to the contents of the three required search buttons: “文档” (Document), “图片” (Image), and “媒体” (Media). The content corresponding to the search term can be found in the frame below.

It can be seen that the operation and maintenance of the system is simple and easy to learn, which does not require special programming capability. The spare file archive administrator can start the operation with a little training.

\section{Conclusions}

The system can realize various functions designed, and creatively introduces multimedia and information technology into a simple and convenient information operation, which greatly improves the correct rate and efficiency of retrieval.

At the same time, the application of Python development tools makes the system highly portable and low dependence on hardware performance. It is no longer limited to traditional devices, such as personal computers. Even a smart phone can be used as a running platform. It shows outstanding application scalability and dexterity.

In addition, this open information data management system, which has a good application advantage, can be used not only for the device management work described in the paper, but also for multimedia file material specification 
according to the requirements of various specific information management applications.

\section{Acknowledgements}

At that period, we worked very hard for our goal. Thanks to all ones of our group, we finish this work successfully.

\section{Conflicts of Interest}

The authors declare no conflicts of interest regarding the publication of this paper.

\section{References}

[1] Li, W.-L. and Wei, X.-C. (2014) Research on the Classification of Spare Parts for Supplier Management. Management Science \& Engineering (ICMSE), IEEE, Helsinki, Finland, 17-19 August 2014, 379-386.

[2] Lv, J.R., Zhang, G.B. and Wang, S.H. (2017) Penetration of Cloud Computing Technology in the Informationalization Process of Cold Chain Logistics. 2nd International Conference on Artificial Intelligence and Engineering Applications (AIEA 2017), Wu Han Zhicheng. Co. Press, Guangxi, 209-215.

[3] Casu, F., Manunta, M., Agram, P.S. and Crippen, R.E. (2017) Big Remotely Sensed Data: Tools, Applications and Experiences. Remote Sensing of Environment, 202, 1-2. https://doi.org/10.1016/j.rse.2017.09.013

[4] McComb, C., Cagan, J. and Kotovsky, K. (2017) Data on the Configuration Design of Internet-Connected Home Cooling Systems by Engineering Students. Data in Brief, 14, 773-776. https://doi.org/10.1016/j.dib.2017.08.050

[5] Wang, K., He, F., Zhang, G.Y., Jiang, S. and Gong, D. (2018) Comparative Experimental Analysis on Various Picking Types under Dedicated and Random Storage Assignments for Automated Storage and Retrieval Systems. Journal of Chongqing University (English Edition), 17, 101-118.

[6] Gins, W., de Groote, R.P., Bissell, M.L., Granados Buitrago, C., Ferrer, R., Lynch, K.M., Neyens, G. and Sels, S. (2018) Analysis of Counting Data: Development of the SATLAS Python Package. Computer Physics Communications, 222, 286-294. https://doi.org/10.1016/j.cpc.2017.09.012

[7] Chen, Z.F., Ma, W.W.Y., Lin, W., Chen, L., Li, Y.H. and Xu, B.W. (2018) A Study on the Changes of Dynamic Feature Code When Fixing Bugs: Towards the Benefits and Costs of Python Dynamic Features. Science China (Information Sciences), 61, 169-186. 